\title{
Effect of Cytochalasin B, Lantrunculin B, Colchicine, Cycloheximid, Dimethyl Sulfoxide and Ion Channel Inhibitors on Biospeckle Activity in Apple Tissue
}

\author{
Andrzej Kurenda • Piotr M. Pieczywek • \\ Anna Adamiak • Artur Zdunek
}

Received: 18 February 2013 / Accepted: 3 June 2013 / Published online: 22 June 2013

(C) The Author(s) 2013. This article is published with open access at Springerlink.com

\begin{abstract}
The biospeckle phenomenon is used for nondestructive monitoring the quality of fresh fruits and vegetables, but in the case of plant tissues there is a lack of experimentally confirmed information about the biological origin of the biospeckle activity (BA). As a main sources of $\mathrm{BA}$, processes associated with the movement inside the cell, such as cytoplasmic streaming, organelle movement and intra- and extracellular transport mechanisms, are considered. The aim of this study is to investigate the effect of metabolism inhibitors, connected with intracellular movement such as cytochalasin B, lantrunculin B, colchicine, cycloheximid, dimethyl sulfoxide (DMSO) and mixture of ion channel inhibitors, injected into apples, on BA. Two methods of BA analysis based on cross-correlation coefficient and Laser Speckle Contrast Analysis (LASCA) were used. DMSO, lantrunculin B and mixture of ion channel inhibitors have a significant effect on BA, and approximately $74 \%$ of BA of apple tissue is potentially caused by biological processes. Results indicate that the functioning of actin microfilaments and ion channels significantly affect BA.
\end{abstract}

Key words Apple tissue - Biological processes .

Biospeckle · Image analysis · Intracellular movements · Metabolism inhibitors

\section{Introduction}

Biospeckle is a variable in time and space interference pattern, formed as the result of illumination of the living

A. Kurenda $(\varangle) \cdot$ P. M. Pieczywek $\cdot$ A. Adamiak $\cdot$ A. Zdunek Department of Microstructure and Mechanics of Biomaterials, Institute of Agrophysics, Polish Academy of Sciences, Doswiadczalna 4, 20-290 Lublin 27, Poland

e-mail: a.kurenda@ipan.lublin.pl organism by coherent light. Biospeckle is observed only for biological materials and is a result of the micromovement of internal elements of a biological object. The measure of biospeckle pattern dynamics is biospeckle activity (BA), obtained by various methods of image analysis. At present, biospeckle is studied as a potential tool in medicine, plant biology and agriculture, for example, in real-time, noninvasive examination of microcirculation in different organs of the human body [1], non-destructive evaluation of viability of seeds [2], activity of parasites in living tissues [3], analysis of maturation and bruising of fruits and vegetables [4] and monitoring of apple shelf life [5].

The physics of biospeckle is well-developed [6], but experimentally confirmed information about the biological background of biospeckle of plant tissues are scarce. BA is the result of movements inside a tissue. Three types of processes can, therefore, be taken into account as its source: physical processes (diffusion, Brownian motion, evaporation from the surface of biological object), instability of measuring equipment and biological processes such as: cytoplasmic streaming, organelle movement, transport of substances in the cell, transport of water and solutes in vascular bundles, cell growth and division, etc. [7]. However, there is a lack of experiments explaining which of these processes is the main source of BA.

The goal of this study is to investigate the effects of cytochalasin B, lantrunculin B, colchicine, cycloheximid, dimethyl sulfoxide (DMSO) and a mixture of ion channel inhibitors (ICI) (anthracene-9-carboxylic acid (A9C), tetraethylammonium chloride (TEA), gadolinium chloride and diethylstilbestrol (DES)) on BA. Except DMSO, these substances non-destructively and highly selectively influence processes associated with the movement of cytoplasmic organelles and substances.

Cytochalasin B inhibits polymerization [8] and lantrunculin B causes depolymerization [9] of actin filaments, and both, 
cease the transport associated with the actin cytoskeleton [10] as well as cytoplasmic streaming [11]. Colchicine, binding to tubulin dimers, prevents microtubule polymerization, stopping their reorganization [12]. In turn, cycloheximide - an inhibitor of translation - blocks protein synthesis and can potentially reduce the number of emerging and moving scattering centres in the cytoplasm in the form of the protein [13]. Since changes in intracellular ions concentration alter cytoplasmic streaming [14], an ICI, blocks the transport of $\mathrm{K}^{+}, \mathrm{Ca}^{2+}$, anions and protons [15], and can affect movements of cytoplasm and a number of secondary processes.

\section{Materials and Methods}

Experiments were conducted on apples (Malus domestica Borkh cv. 'Idared') received from The Institute of Horticulture (Skierniewice, Poland). Fruit were harvested in the optimum harvest window based on the ethylene and starch tests, and stored in a cold room at $2{ }^{\circ} \mathrm{C}$ in a normal atmosphere for 4 months. About $24 \mathrm{~h}$ before experiment apples were conditioned at room temperature.

In the experiment, nine different solutions at amounts of 100-200 $\mu \mathrm{l}$ were injected into apple tissues with a $1 \mathrm{ml}$ syringe (Fig. 1):

- SS (Standard Solution) - containing deionized water, $1 \mathrm{mM} \mathrm{KCl}, 1 \mathrm{mM} \mathrm{CaCl}_{2}$ and $50 \mathrm{mM}$ mannitol (pH 7 TRIS/MES) - as a control for the injection method and the base for preparation of solutions of active substances.

- $1 \%$ DMSO (1\% solution of dimethyl sulfoxide in SS, $\mathrm{v} / \mathrm{v}$ ) - as a control of the effect of $1 \% \mathrm{DMSO}$ (used to solve cytochalasin B, lantrunculin B, A9C and DES) on BA.

- MANN (0.5 M solution of mannitol in SS) - as a control of effect of hypertonic solution on BA.

- $\mathrm{CB}(0.5 \mathrm{mM}$ solution of cytochalasin B in $1 \% \mathrm{DMSO})$ to verify the effect of actin microfilaments' polymerization inhibition on BA.

- LANTR (0.5 mM solution of lantrunculin $B$ in $1 \% \mathrm{DMSO}$ ) - to verify the effect of actin microfilaments' depolymerization on BA.

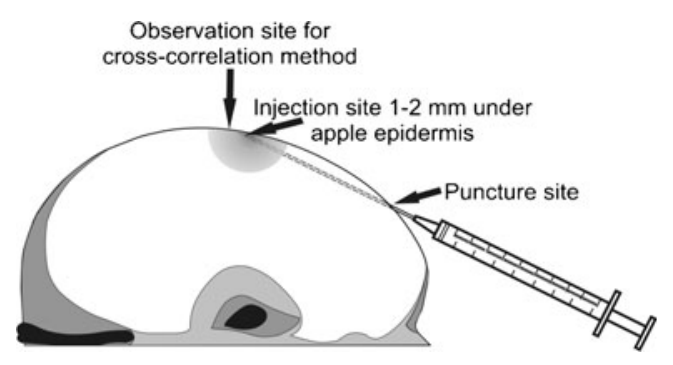

Fig. 1 Schematic presentation of the injection method
- COLCH (2.5 mM solution of colchicine in SS) to verify the effect of microtubules' reorganization inhibition on BA.

- CYCL (1 mM solution of cycloheximid in SS) to verify the effect of the inhibition of protein synthesis on BA.

- ICI (mixture of ion channel inhibitors in 1\%DMSO in $1 \mathrm{mM}$ concentration each: A9C, TEA, DES, gadolinium chloride) to verify the effect of the inhibition of ion transport through the cell membranes on BA.

- $100 \%$ DMSO - to verify the effect of cell destruction on BA.

\section{Biospeckle Activity Measurements}

BA was evaluated by two different systems and approaches. The first method based on cross-correlation coefficient was used to precisely quantify the BA on a small area of apple surface, near to the place of injection. The second method, Laser Speckle Contrast Analysis (LASCA), was used for qualitative evaluation of BA through the creation of maps of the BA of the whole visible side of the fruit.

The system for cross-correlation BA measurements $[5,16$, 17] (Fig. 2) consists of a diode laser ( $30 \mathrm{~mW}, \lambda=635 \mathrm{~nm}$, Newport, USA), with a $20 \times$ laser beam expander (Edmund Optics, USA) to illuminate the sample, and a CCD camera (Monochrome FireWire Astronomy Camera DMK 21AF04.AS, The Imaging Source Europe GmbH, Germany) with a $25 \mathrm{~mm}$ objective and a $20 \mathrm{~mm}$ extension ring to detect scattered light. The distance between camera and object was $37 \mathrm{~mm}$ and that between laser and object was $180 \mathrm{~mm}$. The illumination angle was $30^{\circ}$. Experiment was conducted in a dark room, and the light source in addition to low-intensity laser light was a computer. Expanded laser light intensity registered at distances corresponding to the laser-object in both systems was less than $1 \mu \mathrm{mol} \mathrm{m} \mathrm{m}^{-2}$. Biospeckle activity was recorded as uncompressed movies (AVI, 8 bits, RGB24 codec) lasting $4 \mathrm{~s}$

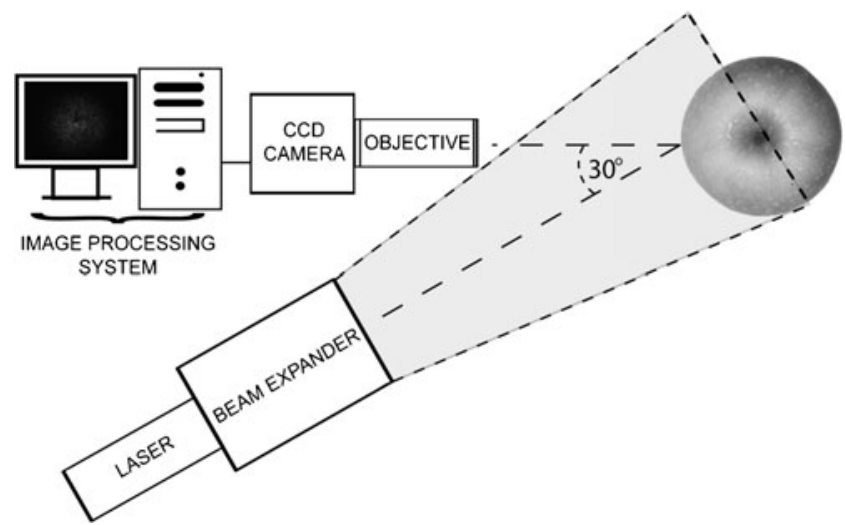

Fig. 2 Scheme of experimental setup for BA measurements (top view) 
with 15 frames per second. The image resolution was $320 \times 240$ pixels, which corresponded to an observation area of $7.5 \mathrm{~mm}^{2}$. The image exposure time was $1 / 250 \mathrm{~s}$. Gain and brightness was found experimentally so as to avoid overexposures of pixels on the image histogram. The image acquisition settings were kept unchanged during the experiment.

BA was determined by means of the "corrcoef" function available in Matlab as a cross-correlation coefficient $\left(\mathrm{C}^{4}\right)$ between speckle frames recorded with $4 \mathrm{~s}$ lag. Then, BA was calculated as $1-\mathrm{C}^{4}$.

The second system for biospeckle measurements using the LASCA method consisted of the same elements (Fig. 2), and the same settings were used as in the cross-correlation method, with the exception that the camera lens had a $5 \mathrm{~mm}$ extension ring, distances from camera to object and from laser to object were $450 \mathrm{~mm} 630 \mathrm{~mm}$, respectively, and a $400 \times$ laser beam expander was used. This allowed observation an area of about $70 \mathrm{~cm}^{2}$, which was enough to capture the whole side of the apple in a single frame.

LASCA is a technique using the modified Briers contrast method, which allows showing the spatial distribution of speckle activity [18]. The LASCA algorithm is based on calculations of spatially or time-averaged contrast over the local square window of pixels. In this study, the contrast value $C_{x, y}$ was calculated for each pixel separately, according to equation:

$C_{x, y}=\frac{\sigma_{x, y}}{I_{x, y}}$

Where $\sigma_{x, y}$ and $I_{x, y}$ are the standard deviation and the mean intensity, respectively. Both values were calculated for pixels in location $x, y$, in 60 frames. A threshold value of mean intensity equal to 5 was used, in order to reduce the influence of camera noise in less illuminated areas of the image. The resulting contrast map was spatially averaged using a $3 \times 3$ pixel size overlapping the square window. This operation was carried out in order to smooth generated images of BA. High contrast values on images obtained using this technique occur in areas with high speckle fluctuations. In areas where there are no fluctuations, the spatial contrast is reduced.

\section{Experimental Procedure}

To determine the effect of solutions of chemicals on BA, 12-24 apples for each solution were tested (Table 1), using both, cross-correlation and LASCA method.

Apples were placed in a measurement setup and recording of the movies began. After 60 min of recording, apples were removed from the setup, appropriate solution under the apple skin was injected (what took about $30 \mathrm{~s}$ ), and apples were placed in the setup again for another $600 \mathrm{~min}$. In the cross-

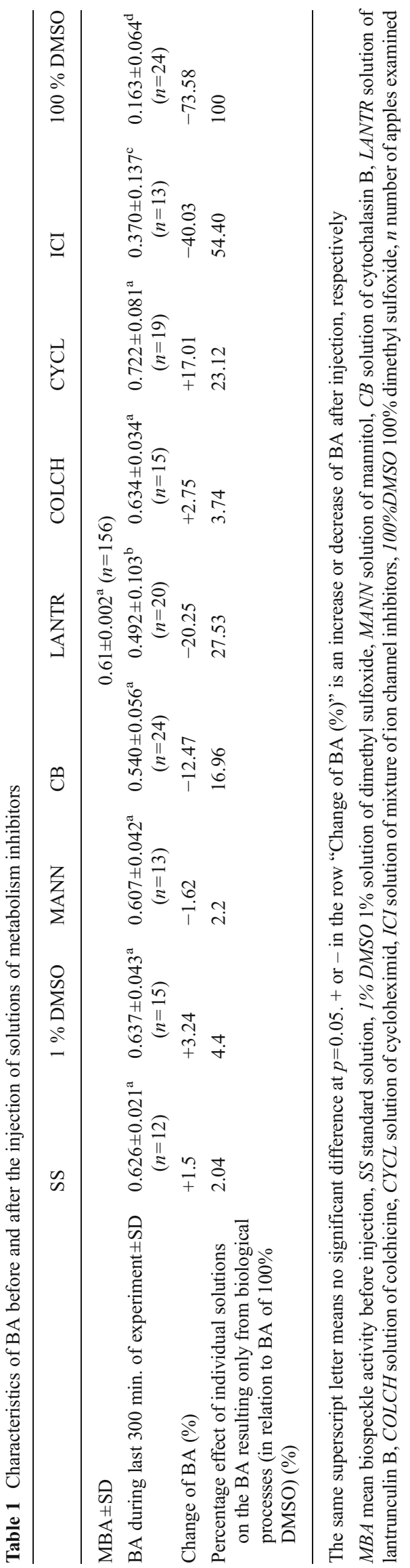


correlation method for each individual fruit, during $660 \mathrm{~min}$ a stack of 330 movies lasting $4 \mathrm{~s}$ each, with an interval of 2 min, were recorded using custom made software. For each apple, whole recording time was divided in three parts for BA calculations. The first $60 \mathrm{~min}$ were considered as the reference for the condition of apples before injection. The second part, the next $300 \mathrm{~min}$, consisted of the injection of solutions and the stabilization of biological processes after injection. The third part, lasting $300 \mathrm{~min}$, was the monitoring of effects which occurred as a result of the injected solutions (Fig. 3). The observation area was set to about 2-3 mm over the place where the solution was injected (Fig. 1). After measurements for the cross-correlation method, one $4 \mathrm{~s} \mathrm{mov-}$ ie for the LASCA method was recorded. After experimentation, tissue samples of $150 \mu \mathrm{m}$ thickness taken exactly from the injection place were cut by VT 1000S Leica vibratome and imaged with a SZX16 Olympus stereoscope in dark field mode.

\section{Statistical Analysis}

Mean values of BA with standard deviations and other statistics were calculated using STASTICA ${ }^{\circledR} 8.0$ (StatSoft, Inc. 1984-2008). One-way ANOVA with Tukey HSD post-hoc was used for checking the effect of injected chemicals on BA. The significance of effects was determined at $p<0.05$.

\section{Results}

Before injection of solutions (Fig. 3, 0-60 min of the experiment), in all cases BA was stable. Mean biospeckle activity (MBA; approximately 0.617) was calculated for all apples before injection and used as a reference for the estimates presented below. BA of apples before injection did not significantly differ.

Just after injection of solutions, indicated in Fig. 3, the BA suddenly increased and its stabilization lasted for at least $60 \mathrm{~min}$. The solution effects were statistically analysed based on the data from the last $300 \mathrm{~min}$ of measurements (Fig. 3, 360-660 min of the experiment), because within the first few hours after injection BA could be affected by stress relaxation in the tissue. In Table 1, averaged values of BA for each solution during the last $300 \mathrm{~min}$ of the experiment are shown. In the case of SS, $1 \%$ DMSO, MANN and COLCH, BA stabilized after injection at a value equal or slightly higher than before injection (Fig. 3). Statistical analysis showed no significant effects of these solutions on BA in comparison to apples before injection. A statistically significant effect was found $(F=45.816, p<0.000)$ after application of $100 \%$ DMSO, ICI and LANTR. A transient but significant effect was observed after CB application (Fig. 3, between 240 and $360 \mathrm{~min}$ of the experiment). The strongest effect was observed after application of $100 \%$ DMSO which caused, during the last $300 \mathrm{~min}$ of the experiment, $\mathrm{BA}$ to decrease by about $74 \%$ of MBA. For ICI and LANTR, BA decreased by about $40 \%$ and $20 \%$ of MBA, respectively (Table 1).

Examples of apples appearance at about $600 \mathrm{~min}$ after injection, biospeckle images, results of LASCA and micrographs of injection place, for SS and solutions for which a significant effect was obtained (LANTR, ICI, $100 \%$ DMSO) are presented in Fig. 4. Apples after injection of solutions, which are not presented in the Fig. 4 are similar to SS. Places of injection are marked by an arrow and black point in row A. One can observe visible bruising around the injection place on the skin of apples (Fig. 4, row A) which appeared
Fig. 3 Biospeckle activity before and after injection of solutions indicated in the legend. Additionally, SP is BA observed on a steel plate

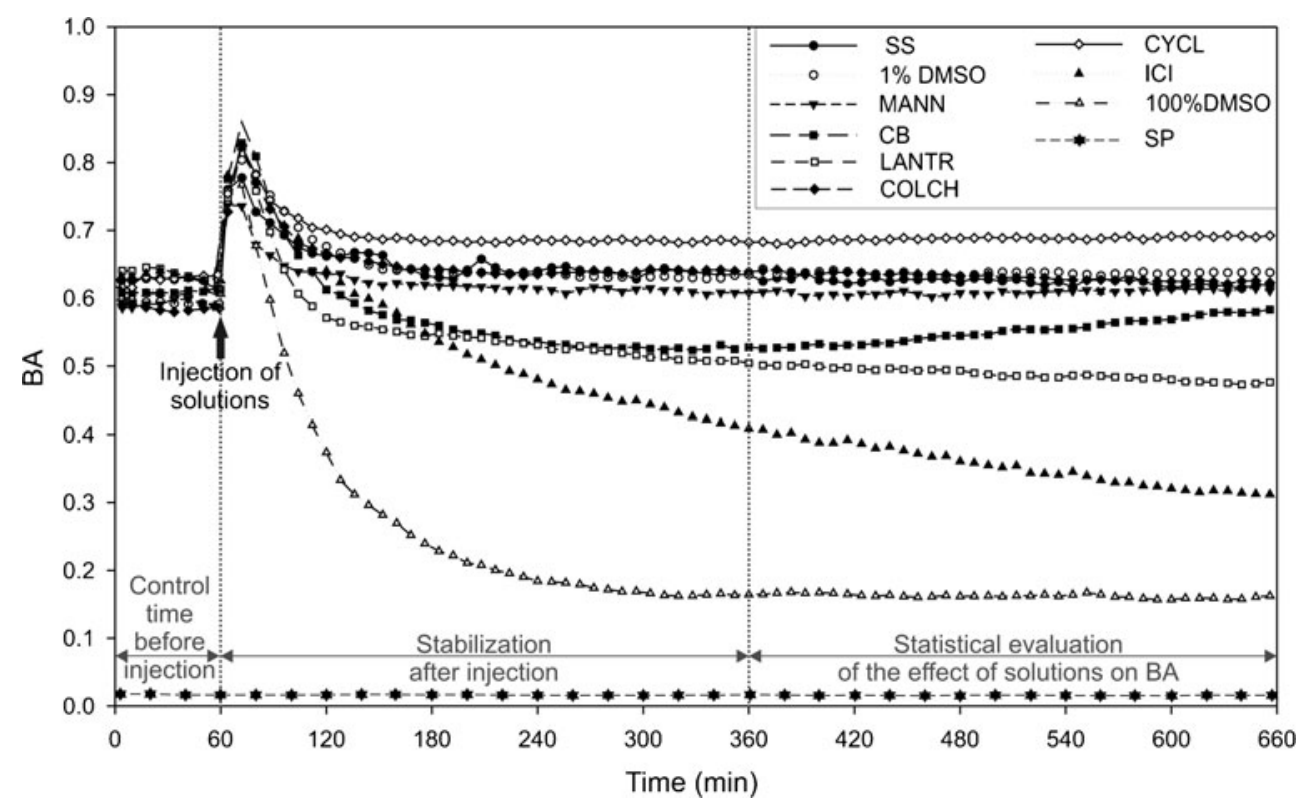






Fig. 4 Examples of: A apples 600 min after injection and just before recording a biospeckle patterns for LASCA, B biospeckle pattern recorded for LASCA analysis. C results of LASCA analysis, D micrographs of apple tissues close to the place of injection. Scale for LASCA

only for $100 \%$ DMSO. LASCA analysis (Fig. 4, row C) showed that areas with low BA near the place of injection, occurred in the case of $100 \%$ DMSO, LANTR and ICI which is consistent with the results of cross-correlation analysis. Micrographs (Fig. 4, row D) revealed that in the case of $100 \%$ DMSO and ICI, in the place of injection, a free space existed between apple epidermis and parenchymatic cells. It was noticed that during the injection of chemicals, in 60 $70 \%$ of cases, blisters appeared in response to fluid pressure, also in the case of SS and LANTR. It was noted that blistering depended on applied pressure and on the properties of fruit tissue. For 30-40\% of apples where the blisters did not appear, injected fluid spread easily in the tissue under the fruit skin, and that was clearly visible on the skin. Additionally, these apples did not differ in terms of BA or appearance a few hours after injection.

\section{Discussion}

Injection or infusion of a solution of chemical substances into the stems of vascular plants is used in plant physiology in situations, when a systemic effect and relatively low tissue damage is needed. This method (Fig. 1) was adapted for the analysis is presented beside (values on the scale are not equal with BA derived from the cross-correlation method). Width of images shown in the rows $\mathbf{A}-\mathbf{C}$ is $5 \mathrm{~cm}$ and in $\mathbf{d} 8 \mathrm{~mm}$

application of chemical factors into apple tissue, to reveal a $\mathrm{BA}$ response to changes of selected biological processes.

Puncturing of fruit epidermis and parenchymatic cells, and application of the fluid under pressure, affected the structure of the tissue in areas where injected fluid reached. This resulted in the formation of blisters and free space under the apple skin (Fig. 4, row D). Injected solutions did not propagate systemically, but remained within a few centimetres of the injection site. However, no browning occurred at the treatment place after application of SS, LANTR, ICI (Fig. 4) and $1 \%$ DMSO, MANN, CB, COLCH, CYCL (not shown), even after prolonged storage following the experiment.

BA before and after injection of control solutions (SS, $1 \%$ DMSO and MANN) was similar (Table 1). It should be noted that just after injection BA suddenly increased, probably due to stress relaxation in the tissue after pressure treatment and the propagation of the injected fluid, but in the case of SS, $1 \%$ DMSO and MANN, BA stabilized over the next $1 \mathrm{~h}$ at a value equal or slightly higher (difference not significant) than before injection. Lack of browning and unchanged BA a few hours after injection of SS, $1 \%$ DMSO and MANN suggests, that the application of hydrostatic pressure only caused cell-to-cell debonding, without disruption of cell 
membranes and the maintenance of cell function. The above observations of tissue appearance and BA before and after injection of control solutions suggest, that the method of injection has no significant effect on the presented results, despite some damages to the structure of tissues.

The results of cross-correlation analysis (Table 1) showed, that the disruption of cell membranes by $100 \%$ DMSO, and the rapid death of cells at the application place, caused a decrease of BA by about $74 \%$ in relation to MBA. Therefore, the BA observed during the last $300 \mathrm{~min}$ of the experiment was probably the result mainly physical phenomena and hardware noises, and can be treated as the lowest BA caused by the functioning of living cells. The remaining $26 \%$ of the BA is the result of hardware noises and physical processes. BA measured on a steel plate (SP) could be used to distinguish $\mathrm{BA}$ from noise (BA for $\mathrm{SP}=0.015$; Fig. 3). The difference between BA obtained after application of $100 \%$ DMSO and SP, is the result only of physical processes inside the tissue and amounted to $24 \%$. According to this calculation BA caused by hardware noises is $2 \%$. Moreover, assuming that $100 \%$ DMSO destroys all living cells near the area of injection, the percentage effect of other substances on the MBA resulting only from biological processes could be calculated (Table 1). If the $74 \%$ decrease in total BA after injection of $100 \%$ DMSO, corresponds to a $100 \%$ stop of biological processes in apple tissue, ICI and LANTR, for which a statistically significant effect was obtained, decreased $\mathrm{BA}$ in relation to the $100 \%$ DMSO by $54 \%$ and $27 \%$, respectively. These two percentages reflect contributions of ICI and LANTR to all biological processes influencing BA.

ICI caused the second major effect on BA (decrease of BA by about $40 \%$ compared to MBA and a $54 \%$ share in BA resulting only from biological processes). The composition of ICI was chosen to inhibit the movement of the major inorganic ions through the cell membrane. The ion fluxes probably did not have a direct impact on the BA, because the size of the ions was too small relative to the wavelength of the laser used. However, multidirectional secondary effects could directly affect the movement of larger organic molecules and organelles, which are considered to be the source biospeckle activity. The combined effect of a reduction in the transport of organic molecules coupled with the transport of ions, the inhibition of conformational changes in carrier proteins, the lack of proper regulation of osmotic pressure in the cell and disruption of cell signalling caused a decrease in BA. The functioning of ion channels is also directly connected with cytoplasmic streaming [19]. During action potentials in Chara and Nitella cells, cytoplasmic streaming stops, which is probably connected with an increase in the concentration of $\mathrm{Ca}^{2+}$ in the first phase of action potential [20] and propagation of information about stimulus by calcium signalling [21], or with the direct effect of divalent ions (especially $\mathrm{Mg}^{+2}$ ) on the actin cytoskeleton [22]. More information on the relation between ion transport and BA, can be obtained in an experiment using single ion channel inhibitors.

Considering contribution of cytoskeleton to the movement in the cell and consequently to apparent BA, the effects of CB, LANTR and COLCH should be included. It was assumed that both, actin filaments and microtubules, are involved in the generation of cellular movement, and the inhibition of main processes connected with these structures (e.g.: reconstruction of the cytoskeleton, cytoplasmic streaming, transport substances or organelle positioning) will reduce BA. However, the results of experiments showed, that only substances connected with inhibition of actin filaments (CB, LANTR) decreased BA, while in the case of COLCH, BA only slightly increased. In the case of COLCH it should be taken into account, that the lack of effect can also be the result of colchicine's low affinity to apple tubulin [23] or low variability microtubules in apple cells. In conclusion, only the inhibition of functioning of the actin part of cytoskeleton affected movement inside the cells, which resulted in a significant decrease of BA (decrease of BA after injection of LANTR by about $20 \%$ compared to MBA and a $27 \%$ share in BA resulting only from biological processes).

Inhibition of protein synthesis at ribosomal stage by CYCL was expected to decrease BA due to a decrease in the number of scattering centres in the cytoplasm in the form of proteins and protein transport vesicles. However, the results of this experiment indicated the opposite, an increase tendency of BA was noted by about $17 \%$ (not significant). This may be caused by starting additional or an increase in the existing rate of cellular mechanisms or by an increase in the amino acid content [24], which also could speeds up biospeckle formation.

Information obtained by cross-correlation analysis are confirmed by LASCA (Fig. 4), which allows for spatial analysis of BA. In the case of $100 \%$ DMSO, ICI and LANTR, around the area of injection, a clear decrease of BA was visible, while for other solutions the areas with decreased activity were not present.

In the light of the information presented above, it is possible to discuss practical application of the biospeckle phenomenon in fields of food science and biology. Cellular processes as cytoskeleton reconstruction, cytoplasmic streaming and ionic fluxes between cellular compartments and through the cell membrane, that have the greatest impact on $\mathrm{BA}$, are changeable during plant development, maturation and aging as well as may be disrupted as a result of mechanical damage or pathogen attack. At present stage of advancement of methods of biospeckle image analysis, biospeckle techniques could be applied to determination the general rate of metabolism during the fruits life and for creation of spatial maps of BA of the surface of the whole fruits, which may be useful for early detection and visualization of mechanical 
damages and infections. Sensitivity of biospeckle to vibration does not allow the use on production lines, but potentially can be used in long-term monitoring of the metabolic state and health of the agricultural products, for example during shelf life or cold storage.

In addition to vibration, use of biospeckle techniques is currently also limited due to inability to distinguish the dynamics generated by specific metabolic of physical process in the overall biospeckle signal. The solution of this problems may be the development of new measurement methods based on frequency analysis, which can open new possibilities for the study of specific physiological processes in the green parts of plants. An examples of potential application of biospeckle in this case, could be the study of fruits or leaves physiology, where, non-destructively, cell wall and cytoskeleton reconstruction, cytoplasmic streaming or water status during development and aging could be measurable. Thus, at present, applications of biospeckle for fruits quality evaluation are possible, but wider application in fields of food technology and plant physiology require more specific model and theoretical studies.

\section{Conclusions}

In summary, this study presents the evidence that approximately $74 \%$ of BA observed in apple tissue is potentially caused by the life processes. Action of the actin part of cytoskeleton and processes connected with functioning of ion channels and proton pumps make a significant contribution to the total BA. Obtained information indicate that biospeckle, as a simple and non-destructive technique, can be developed toward monitoring not only fresh produce quality but also specific biological phenomena like cytoplasmic streaming or intercellular transport.

Acknowledgments The study was supported by The National Centre for Research and Development: NR 12013710.

Open Access This article is distributed under the terms of the Creative Commons Attribution License which permits any use, distribution, and reproduction in any medium, provided the original author(s) and the source are credited.

\section{References}

1. J.D. Briers, Laser Doppler, speckle and related techniques for blood perfusion mapping and imaging. Physiol. Meas. 22(4), R35-R66 (2001)

2. G.H. Sendra, R. Arizaga, H.J. Rabal, M. Trivi, Decomposition of biospeckle images in temporary spectral bands. Opt. Lett. 30(13), 1641-1643 (2005)
3. R.A. Braga, G.F. Rabelo, L.R. Granato, E.F. Santos, J.C. Machado, R. Arizaga, H.J. Rabal, M. Trivi, Detection of fungi in beans by the laser biospeckle technique. Biosyst. Eng. 91, 465-469 (2005)

4. G.F. Rabelo, R.A. Braga Jr., I.M.D. Fabbro, R. Arizaga, H.J. Rabal, M.R. Trivi, Laser speckle techniques applied to study quality of fruits. Rev. Bras. Eng. Agric. Ambient 9, 570-575 (2005)

5. A. Zdunek, L. Muravsky, L. Frankevych, K. Konstankiewicz, New nondestructive method based on spatial-temporal speckle correlation technique for evaluation of apples quality during shelf-life. Int. Agrophys. 21, 305-310 (2007)

6. J.C. Dainty, Laser speckle and related phenomena (Springer, Berlin, 1975)

7. R.A. Braga, L. Dupuy, M. Pasqual, R.R. Cardosos, Live biospeckle laser imaging of root tissues. Eur. Biophys. J. 38, 679-686 (2009)

8. D.W. Goddette, C. Frieden, Actin polymerization. The mechanism of action of cytochalasin D. J. Biol. Chem. 261(34), 15974-15980 (1986)

9. W.M. Morton, K.R. Ayscough, P.J. McLaughlin, Latrunculin alters the actin-monomer subunit interface to prevent polymerization. Nat. Cell Biol. 2(6), 376-378 (2000)

10. V.R. Simon, L.A. Pon, Actin-based organelle movement. Experientia 52(12), 1117-1122 (1986)

11. T. Shimmen, M. Tazawa, Control of cytoplasmic streaming by ATP, $\mathrm{Mg}^{2+}$ and cytochalasin $\mathrm{B}$ in permeabilized Characeae cell. Protoplasma 115, 18-24 (1983)

12. L.G. Bergen, G.G. Borisy, Tubulin-colchicine complex inhibits microtubule elongation at both plus and minus end. J. Biol. Chem. 258(7), 4190-4194 (1983)

13. R.J. Ellis, I.R. MacDonald, Specificity of cycloheximide in higher plant systems. Plant Physiol. 46, 227-232 (1970)

14. T. Shimmen, M. Kikuyama, M. Tazawa, Studies on cessation of cytoplasmic streaming under $\mathrm{K}^{+}$-induced depolarization in Nitella axilliformis. J. Plant Res. 108(4), 457-462 (1995)

15. E. Krol, K. Trebacz, Ways of ion channel gating in plant cells. Ann. Bot. 86, 449-469 (2000)

16. A. Zdunek, W.B. Herppich, Relation of biospeckle activity with chlorophyll content in apples. Postharvest Biol. Technol. 64, 58-63 (2012)

17. A. Kurenda, A. Adamiak, A. Zdunek, Temperature effect on apple biospeckle activity evaluated with different indices. Postharvest Biol. Technol. 67, 118-123 (2012)

18. J.D. Briers, S. Webster, Laser speckle contrast analysis (LASCA): a non-scanning, full-field technique for monitoring capillary blood flow. Biomed. Opt. 1, 174-179 (1996)

19. O. Babourina, K. Voltchanskii, I. Newman, Ion flux interaction with cytoplasmic streaming in branchlets of Chara australis. J. Exp. Bot. 55(408), 2505-2512 (2004)

20. K. Trębacz, W. Simonis, G. Schonknecht, Cytoplasmic $\mathrm{Ca}^{2+}, \mathrm{K}^{+}, \mathrm{CI}^{-}$, and $\mathrm{NO}^{3-}$ activities in the Liverwort Conocephalum conicum L. at rest and during action potentials. Plant Physiol. 106, 1073-1084 (1994)

21. B.K. Drøbak, V.E. Franklin-Tong, C.J. Staiger, The role of the actin cytoskeleton in plant cell signaling. New Phytol. 163, 13-30 (2004)

22. K.V. Thimann, R. Biradivolu, Actin and the elongation of plant cells. II. The role of divalent ions. Protoplasma 183, 5-9 (1994)

23. L.C. Morejohn, T.E. Bureau, L.P. Tocchi, D.E. Fosket, Resistance of Rosa microtubule polymerization to colchicine results from a low-affinity interaction of colchicine and tubulin. Planta 170, 230 241 (1987)

24. J.S. Fletcher, H. Beevers, Influence of cycloheximide on the synthesis and utilization of amino acids in suspension cultures. Plant Physiol. 48, 261-264 (1971) 